\title{
Nanosilver-Decorated Biodegradable Mesoporous Organosilica Nanoparticles for GSH-Responsive Gentamicin Release and Synergistic Treatment of Antibiotic-Resistant Bacteria
}

\author{
Haijun Li, ',* Dongbei Li, ${ }^{2, *}$ \\ Fangman Chen, ${ }^{3}$ Chao Yang, ${ }^{3}$ \\ Xiaogai Li,' Yuan Zhang,' \\ Chunlan Hua, ' Xiaoxu Ma, 'Xin \\ Zhao,' Dan Shao, (D) ${ }^{3}$ Yingshuai \\ Wang, (D) ${ }^{4}$ Liang Ming' \\ 'Department of Clinical Laboratory, The \\ First Affiliated Hospital of Zhengzhou \\ University, Zhengzhou, Henan, 450052, \\ People's Republic of China; ${ }^{2}$ Department \\ of Hematology, Affiliated Tumor Hospital \\ of Zhengzhou University, Henan Cancer \\ Hospital, Zhengzhou, Henan, 450000, \\ People's Republic of China; ${ }^{3}$ Institutes for \\ Life Sciences, School of Biomedical \\ Sciences and Engineering, Guangzhou \\ International Campus, South China \\ University of Technology, Guangzhou, \\ 510630, People's Republic of China; \\ ${ }^{4}$ School of Life Science and Technology, \\ Weifang Medical University, Weifang, \\ Shandong, 26I053, People's Republic of \\ China
}

*These authors contributed equally to this work

Correspondence: Yingshuai Wang School of Life Science and Technology, Weifang Medical University, Weifang, Shandong, 261053, People's Republic of China

Email yingshuaiwang1987@I63.com

Liang Ming

Department of Clinical Laboratory, The First Affiliated Hospital of Zhengzhou

University, Zhengzhou, Henan, 450052,

People's Republic of China

Email mingliang3072@I63.com
Purpose: Antibiotic-resistant bacteria are pathogens that have emerged as a serious public health risk. Thus, there is an urgent need to develop a new generation of anti-bacterial materials to kill antibiotic-resistant bacteria.

Methods: Nanosilver-decorated mesoporous organosilica nanoparticles (Ag-MONs) were fabricated for co-delivery of gentamicin (GEN) and nanosilver. After investigating the glutathione (GSH)-responsive matrix degradation and controlled release of both GEN and silver ions, the anti-bacterial activities of Ag-MONs@GEN were systematically determined against several antibiotic-susceptible and antibiotic-resistant bacteria including Escherichia coli, Pseudomonas aeruginosa, Staphylococcus aureus, and Enterococcus faecalis. Furthermore, the cytotoxic profiles of Ag-MONs@GEN were evaluated.

Results: The GEN-loaded nanoplatform (Ag-MONs@GEN) showed glutathione-responsive matrix degradation, resulting in the simultaneous controlled release of GEN and silver ions. Ag-MONs@GEN exhibited excellent anti-bacterial activities than Ag-MONs and GEN alone via inducing ROS generation, especially enhancing synergetic effects against four antibioticresistant bacteria including Escherichia coli, Pseudomonas aeruginosa, Staphylococcus aureus, and Enterococcus faecalis. Moreover, the $\mathrm{IC}_{50}$ values of Ag-MONs@GEN in L929 and HUVECs cells were $313.6 \pm 15.9$ and $295.7 \pm 12.3 \mu \mathrm{g} / \mathrm{mL}$, respectively, which were much higher than their corresponding minimum inhibitory concentration (MIC) and minimum bactericidal concentration (MBC) values.

Conclusion: Our study advanced the development of Ag-MONs@GEN for the synergistic and safe treatment of antibiotic-resistant bacteria.

Keywords: mesoporous organosilica nanoparticles, nanosilver, gentamicin, GSH-responsive release, antibiotics-resistant bacteria

\section{Introduction}

Antibiotic-resistant bacteria are emerging pathogens that represent one of the dominant challenges in human health, as they reduce the efficacy of conventional antibiotics and significantly increase the number of clinical cases of severe infection. ${ }^{1,2}$ To address this challenge, efforts have been made to develop alternative strategies and anti-bacterial therapeutics based on sophisticated materials to treat antibiotic-resistant bacteria. ${ }^{3-5}$ Recently, nanoparticles (NPs) have emerged as new tools that can be used to not only combat deadly bacterial infections but also 
kill antibiotic-resistant bacteria. ${ }^{6-8}$ Among several metalbased NPs, nanosilver is the most common material used in consumer products and medical equipment. ${ }^{9,10}$ Due to the broad spectrum and long-term activity, nanosilver is an effective antibacterial agent against various bacteria, fungi, and viruses, particularly in killing antibiotic-resistant bacteria. $^{11}$

Current developments have gently shifted attention from monotherapy to combined or multiple therapies to kill antibiotic-resistant bacteria since the synergy of therapeutic agents or techniques give rise to ostentatious superadditive (namely " $1+1>2$ ") therapeutic effects. ${ }^{12}$ There is growing evidence that the combination of nanosilver and antibiotics can produce significant synergistic effects in killing drugresistant bacterial strains due to the overproduction of reactive oxygen species by nanosilver under the influence of antibiotics, thereby expanding the anti-bacterial spectrum of the existing antibiotics. ${ }^{13-15}$ For example, gentamicin (GEN) and other aminoglycoside antibiotics may form chemical complexes with silver ions via binding of the active hydroxyl or amine group. ${ }^{16}$ Thus, scientists proposed a new type of anti-bacterial agent by utilizing the synergistic effects of nanosilver and GEN. ${ }^{17,18}$ However, the antibacterial efficiency of nanosilver and antibiotics may be limited by the off-targeted administration and side effects. Therefore, tailored nanomaterials that integrate nanosilver and antibiotics for "on-demand" drug release would be a more promising and safe system to treat antibioticresistant bacteria.

Nanosilver-decorated mesoporous silica particles (AgMSNs) have been used as a nanosilver-carrying carrier, which protected nanosilver from aggregation and released silver ions in a controlled manner, leading to effective long-term efficacy. ${ }^{19-27}$ However, the uncontrolled biodegradation behavior and silver ions release manner impede the further clinical translation of Ag-MSN. As an alternative, mesoporous organosilica NPs (MONs) were employed to achieve stimuli-responsive degradation and drug release via employing stimulus-responsive cleavable bonds into the silica framework. ${ }^{28-38}$ Our group developed a facile method to fabricate nanosilver-decorated biodegradable MONs (Ag-MONs) with matrix-degradation property, leading to controlled silver ions release against bacteria $^{39}$ With these findings in mind, we hypothesized that Ag-MONs may be selected as an ideal system for GEN delivery, which might achieve synergistic activity against resistant bacteria and less side effects via stimuliresponsive drug release.
In this study, we prepared and characterized nanosilver-decorated biodegradable MONs for GEN delivery against antibiotic-resistant bacteria. After investigating the glutathione (GSH)-responsive matrix degradation and controlled release of both GEN and silver ions, the antibacterial activities of Ag-MONs@GEN were systematically determined against several antibiotic-susceptible and antibiotic-resistant bacteria including Escherichia coli, Pseudomonas aeruginosa, Staphylococcus aureus, and Enterococcus faecalis. Furthermore, the cytotoxic profiles of Ag-MONs@GEN were evaluated.

\section{Experimental Materials}

Tannic acid (TA, 99\%), ammonium hydroxide $\left(\mathrm{NH}_{4} \mathrm{OH}\right.$, $28 \%$ ), tetraethyl orthosilicate (TEOS), and bis[3-(triethoxysilyl)propyl]-tetrasulfide (BTES) were purchased from Sigma-Aldrich Co. (St. Louis, MO, USA). Silver nitrate (AgNO3, 99.5\%) was purchased from Aladdin (Shanghai, China). 3-(4,5-Dimethylthiazol-2-yl)-2,5-diphenyl tetrazolium bromide (MTT), fetal bovine serum (FBS), penicillin, streptomycin, lysogenic broth agar, Dulbecco's modified Eagle medium (DMEM), and lysogenic broth were obtained from Sangon Biotech (Shanghai, China). GEN (> $590 \mathrm{IU} / \mathrm{mg}$ ) was purchased from Dalian Meilun Biotech Co., Ltd. (Dalian, China). All reagents were used without further purification. Deionized water was used in all experiments.

\section{Synthesis of Ag-MONs}

The synthesis of Ag-MONs was conducted according to our previously reported method. ${ }^{39}$ First, TA $(0.5 \mathrm{~g})$ and $\mathrm{NH}_{4} \mathrm{OH}(28 \%, 25 \mathrm{~mL})$ were added into deionized water (200 mL) stirred for $1 \mathrm{~h}$ at room temperature. Next, a mixture of TEOS (100 mg) and BTES (20 mg) was added quickly and stirred for another $2 \mathrm{~h}$. The products were centrifuged and re-dispersed in $\mathrm{AgNO}_{3}$ solution (400 mL, $0.5 \mathrm{mg} / \mathrm{mL}$ ), and vigorous stirring was continued for $4 \mathrm{~h}$ in the dark. Finally, Ag-MONs were collected, washed with ethanol and water, and vacuum-dried.

\section{Characterization}

Transmission electron microscopy (TEM) was done using JEOL ARM-300F. Aqueous solution containing AgMONs $(0.1 \mathrm{mg} / \mathrm{mL})$ was dropped on a copper grid and then dried at room temperature before being observed on TEM. Scanning electron microscope (SEM) images were 
obtained on Hitachi S-4800 to observe the surface morphologies at the acceleration voltage of $5-30 \mathrm{kV}$. $\mathrm{X}$-ray diffraction patterns were performed on the Rigaku D/MAX-2550V diffractometer with $\mathrm{Cu} \mathrm{K} \alpha$ radiation $(\lambda=$ $1.5405 \AA$ ), scanning in the $2 \theta$ ranges of $10^{\circ}-50^{\circ}$. Dynamic light scattering (DLS) measurement was conducted on the Zeta-sizer Nano ZS90. The samples were dispersed in the water. Nitrogen adsorption-desorption isotherms at 77 $\mathrm{K}$ were measured using the Micromeritics TriStar 3000 system. The sample was dried for $5 \mathrm{~h}$ at $120^{\circ} \mathrm{C}$ before measurement. Surface areas were calculated by the Brunauer-Emmett-Teller method. The loading content of GEN and drug release were quantified by HPLC. The mobile phase of HPLC consisted of 5\% ultrapure water and $95 \%$ acetonitrile. The flow rate was $1.0 \mathrm{~mL} / \mathrm{min}$, and column temperature was $25^{\circ} \mathrm{C}$. The contents of $\mathrm{S}$ and $\mathrm{Ag}$ were measured by inductively coupled plasma mass spectrometry (ICP-MS).

\section{Loading and Drug-Release Profile of Ag-MON@GEN}

Ag-MONs (50 mg) were mixed with GEN (25 mL, $2 \mathrm{mg} /$ $\mathrm{mL}$ ) solution, stirred for $4 \mathrm{~h}$, and shaken at a speed of $200 \mathrm{rpm}$ on $37^{\circ} \mathrm{C}$. GEN-loaded Ag-MON (Ag-MON (a) GEN) was collected by centrifugation. To evaluate the GEN-loading capacity, was collected, and residual GEN content in the supernatant was measured by HPLC. In vitro release experiments were done in phosphatebuffered saline (PBS) $(\mathrm{pH}, 7.4)$ with or without $10-\mathrm{mM}$ GSH at $37^{\circ} \mathrm{C}$. Ag-MON@GEN (5 mg) was suspended in $20 \mathrm{~mL}$ PBS (with or without 10-mM GSH) and shaken at a speed of $180 \mathrm{rpm}$ at $37^{\circ} \mathrm{C}$. The amounts of $\mathrm{Ag}$ and GEN released were monitored by ICP-MS and HPLC at different time intervals.

\section{In vitro Antibacterial Assays}

Antibiotic-susceptible and -resistant E. coli, P. aeruginosa, $S$. aureus, and E. faecalis were used as model Gram (-) and Gram (+) bacterial cell strains. The population density of the bacterial cells was determined by measuring the absorbance at $600 \mathrm{~nm}$. In a typical antibacterial assay,

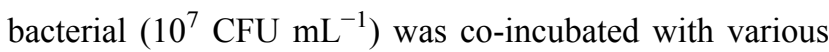
concentrations of Ag-MON@GEN (0, 1.5, 3, 6, 12, 24, and $48 \mu \mathrm{g} / \mathrm{mL}$ ) in Luria-Bertani liquid medium at $37^{\circ} \mathrm{C}$. At each interval, the population density of the bacterial cells was measured, the inhibition curve was plotted.
The minimum inhibitory concentration (MIC) was measured via microbroth dilution method for $16 \mathrm{~h}$ at $37^{\circ} \mathrm{C}$. Ag-MONs@GEN $(0,3,6,12,24$, and $48 \mu \mathrm{g} / \mathrm{mL})$, Ag-MONs (0, 3, 6, 12, 24, 48, 96, and $192 \mu \mathrm{g} / \mathrm{mL})$, and $\operatorname{GEN}(0,1,2,4,8,16,32,64,128$, and $256 \mu \mathrm{g} / \mathrm{mL})$ were separately added into 96 -well plate containing $200 \mathrm{~mL}$ of bacterial cultures and shaken under appropriate conditions. At different time intervals, the optical density (OD) value was detected at $600 \mathrm{~nm}$.

Ag-MONs@GEN $(0,3,6,12,24$, and $48 \mu \mathrm{g} / \mathrm{mL})$, AgMONs $(0,3,6,12,24,48,96$, and $192 \mu \mathrm{g} / \mathrm{mL})$, and GEN $(0,1,2,4,8,16,32,64,128$, and $256 \mu \mathrm{g} / \mathrm{mL})$ were mixed with $1 \times 105 \mathrm{CFU} / \mathrm{mL}$ of bacteria in LB-agar medium. Then, a total of $200 \mu \mathrm{L}$ of bacteria-NP medium was plated on the LB agar plates. The bacteria were incubated at $37^{\circ} \mathrm{C}$ for $24 \mathrm{~h}$ and digital images of each plate were captured. The minimum bactericidal concentration (MBC) was calculated according to the observed colonies in each plate.

\section{Intracellular ROS Generation}

To detect the generation of ROS in, the H2DCFDA probe was used. First, drug-resistant E. coli $\left(10^{6} \mathrm{CFU} / \mathrm{mL}, 100\right.$ $\mu \mathrm{L}$ ) were seeded in a 96-well plate. After $3 \mathrm{~h}$, the medium $(100 \mu \mathrm{L})$ containing Ag-MON@GEN, Ag-MON, MON, or GEN with/without Vitamin C (Vc) was added. After culturing for another $3 \mathrm{~h}$, the H2DCFDA $(10 \mu \mathrm{M})$ was added for $0.5 \mathrm{~h}$. Finally, fluorescence intensity of probe in E. coli was determined.

\section{Statistical Analysis}

Data are expressed as the mean \pm standard deviation. Differences between groups were analyzed using a Student's $t$-test when comparing only two groups. Differences among more than two groups were analyzed using one-way analysis of variance, and the Bonferroni post hoc test was used to analyze the differences between any two groups. $\mathrm{P}<0.05$ was considered statistically significant.

\section{Results and Discussion}

We prepared disulfide-bridged Ag-MONs according to our previously reported one-pot method with some modifications. In this sol-gel process, tannic acid is not only a nonsurfactant template for the formation of MONs but also a reductant for the formation of nanosilver. As shown in representative SEM and TEM images (Figures S1A and B), the as-prepared Ag-MONs exhibited a monodispersed spherical morphology and porous structure with an average 
diameter of about $200 \mathrm{~nm}$. The nanosilver $(2-8 \mathrm{~nm})$ was homogeneously distributed on the surfaces with a mass fraction of $5.58 \%$. Given that the disulfide bond can be cleaved under a high redox intracellular environment of bacteria, our disulfide-bridged Ag-MONs were degraded to small fragments and nanosilver particles after 3 days of incubation in 10-mM GSH solution (Figure S1C-D). Their redox-sensitive matrix degradation might achieve ondemand drug release in response to high GSH levels in bacteria and infections. As shown in Figure S2A, the adsorption peak of Ag-MONs was located at $420 \mathrm{~nm}$, which is attributed to the surface plasmon resonance of nanosilver. The mesoporous characteristics of Ag-MONs have been confirmed by nitrogen sorption isotherm, which exhibited a classic type IV isotherm (Figure S2B). The surface area, pore volume, and average pore diameter of AgMONs were calculated as $378.3 \mathrm{~m}^{2} \mathrm{~g}^{-1}, 0.30 \mathrm{~cm}^{3} \mathrm{~g}^{-1}$, and 4.6 $\mathrm{nm}$, respectively, indicating that they have the promising potential to load sufficient antibiotics.

GEN, a US Food and Drug Administration-approved aminoglycoside antibiotic with a wide range of activities against bacteria, especially Gram-negative bacteria, was selected as a model drug in this study. ${ }^{40}$ GEN has many amino groups, which were loaded on the pores and surface of negatively charged Ag-MONs via electrostatic interactions (Figure 1A). The obtained Ag-MONs @GEN did not show any difference from Ag-MONs in morphology (Figure 1B). After the GEN loading, the surface area, pore volume, and average pore diameter of Ag-MONs@GEN were calculated as $154.3 \mathrm{~m}^{2} \mathrm{~g}^{-1}$,

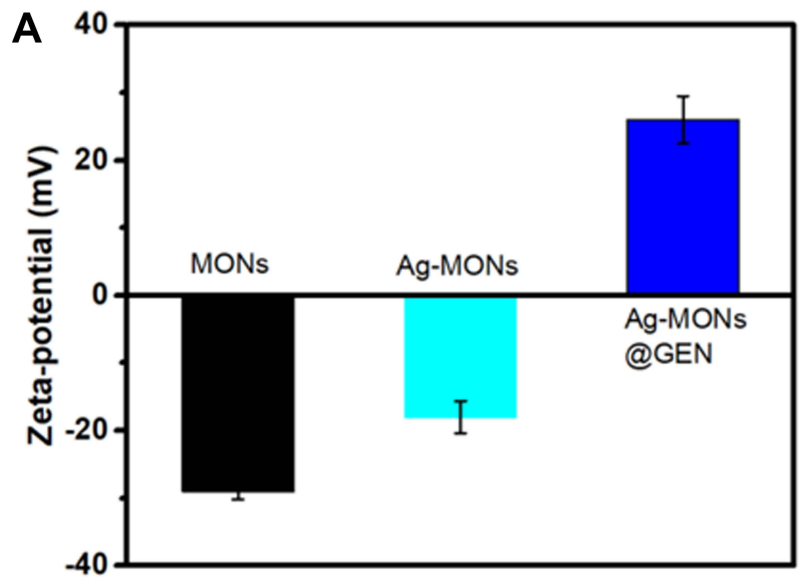

B

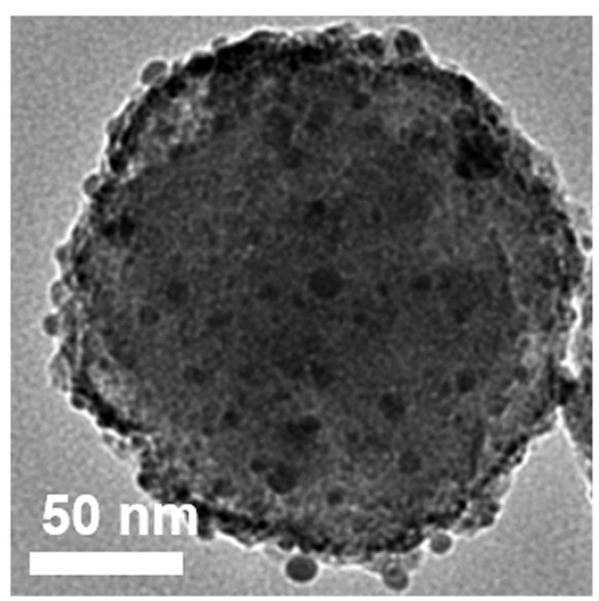

C

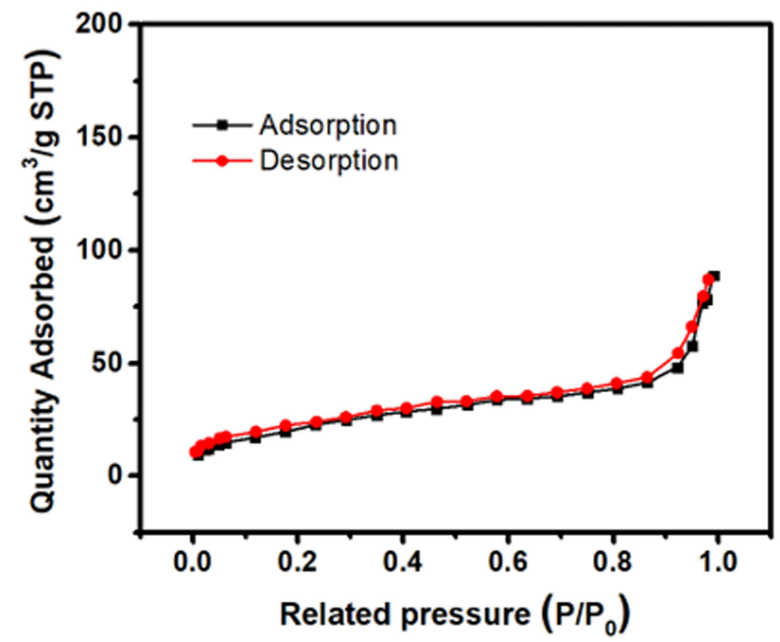

D

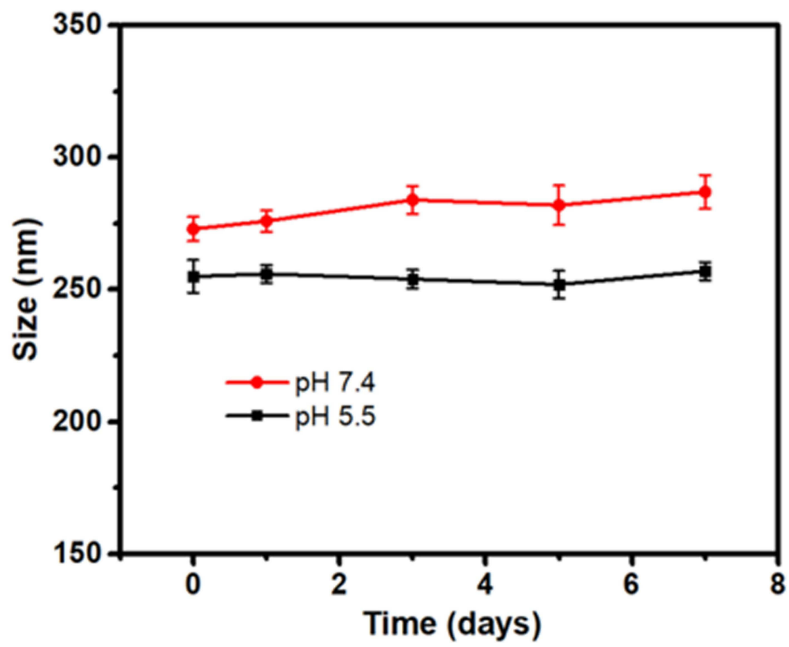

Figure I Characterization of Ag-MONs@GEN.

Notes: (A) Zeta-potential of MONs, Ag-MONs, and Ag-MONs@GEN. (B) TEM image of Ag-MONs@GEN. (C) Nitrogen adsorption-desorption isotherms of Ag-MONs @GEN. (D) The stability of Ag-MONs@GEN in both neutral and acid solutions.

Abbreviations: MONs, mesoporous organosilica nanoparticles; Ag-MONs, nanosilver-decorated mesoporous organosilica nanoparticles; Ag-MONs@GEN, gentamicinloaded nanosilver-decorated mesoporous organosilica nanoparticles; TEM, transmission electron microscopy. 
$0.132 \mathrm{~cm}^{3} \mathrm{~g}^{-1}$, and $1.68 \mathrm{~nm}$, respectively, which were lower than those of the Ag-MONs (Figure 1C). Moreover, the Ag-MONs@GEN was stable in both neutral and acid solutions (Figure 1D). As displayed by HPLC, the loading efficiency and drug-loading content of GEN in the Ag-MONs were determined to be $75.3 \%$ $\pm 3.1 \%$ and $15.2 \% \pm 1.2 \%$, respectively. The in vitro release profiles of GEN and silver ions from Ag-MONs (a)GEN were investigated in PBS with or without 10$\mathrm{mM} \mathrm{GSH}$ at $37^{\circ} \mathrm{C}$. As shown in Figure 2A, the release profiles of GEN showed quick release during the first $24 \mathrm{~h}$, reaching $51.7 \%$ of the loaded GEN amount for Ag-MONs@GEN in 10-mM GSH condition, while only $16.2 \%$ of GEN was released at $0-\mathrm{mM} \mathrm{GSH}$ condition. In the subsequent stage, prolonged release profiles over 72 $\mathrm{h}$ were observed, with a cumulative percentage of $75.6 \%$ at 96 h for Ag-MONs@GEN. As shown in Figure 2B, silver ions were continuously released by Ag-MONs (a) GEN during $96 \mathrm{~h}$ incubation. Similarly, in the presence of 10-mM GSH instead of 0-mM GSH, silver ions of Ag-MONs were released more at the same time, consistent with a previous report showing that the release mode is dependent on GSH. We ascribed this to the fact that disulfide-bridged silica matrix degrades under the GSH condition. Collectively, Ag-MONs (a)GEN showed matrix degradation-controlled dual GEN and silver ion release behavior in response to GSH. Moreover, SEM images showed the

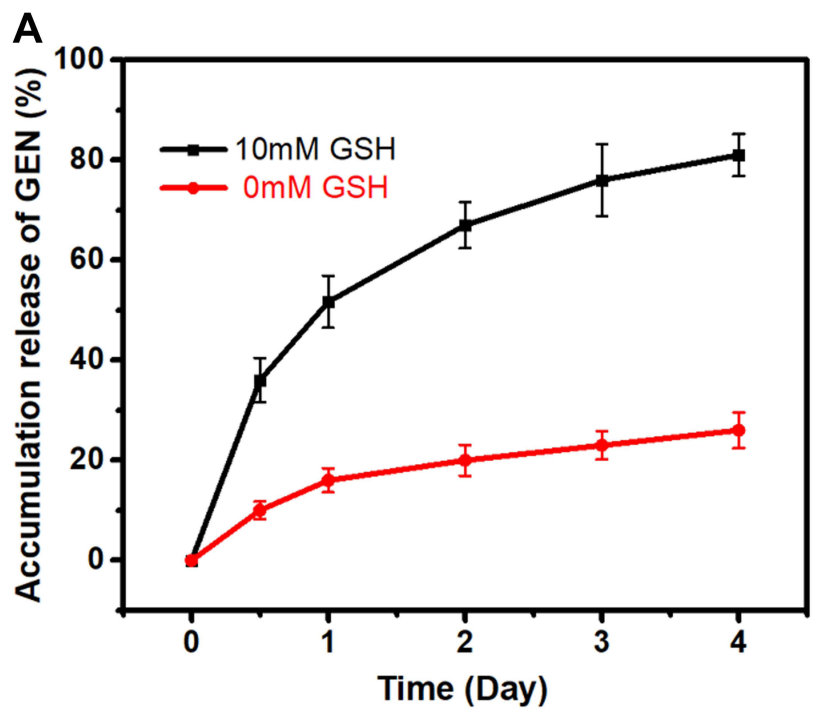

morphological changes in bacteria after the treatment of Ag-MONs@GEN (Figure S3).

To evaluate the in vitro antibacterial activity of AgMONs@GEN, Ag-MONs, and GEN, we selected Gramnegative bacteria E. coli, P. aeruginosa, and Gram-positive bacteria $S$. aureus, E. faecalis as well as their corresponding antibiotic-resistant clinical strains as models. The timerelated effect of optical density values of Ag-MONs @GEN against the growth of antibiotic-susceptible or resistant E. coli, P. aeruginosa, S. aureus, and E. faecalis strains is shown in Figure 3. The results showed that AgMONs@GEN significantly inhibited the growth of all bacterial strains in a dose-dependent manner. The corresponding MIC values of Ag-MONs@GEN, Ag-MONs, and GEN in eight bacterial strains are summarized in Table 1. For Ag-MONs@GEN, the corresponding MIC values in sensitive/resistant $E$. coli, $P$. aeruginosa, S. aureus, and E. faecalis were 3/12, 6/12, 6/24, and 24/ $48 \mu \mathrm{g} / \mathrm{mL}$, respectively. Growth inhibition was more noticeable for Gram-negative bacteria than for Grampositive bacteria because the selective killing effects of both GEN and nanosilver on Gram-negative bacteria have been widely demonstrated. Importantly, the MIC value of Ag-MONs@GEN was lower than that of Ag-MONs and GEN alone in all bacterial strains, especially in the antibiotic-resistant strains. It is worth noting that Ag-MONs @GEN exhibited lower MIC when compared with GEN and Ag-MON, respectively (Table 1). Together, our

B

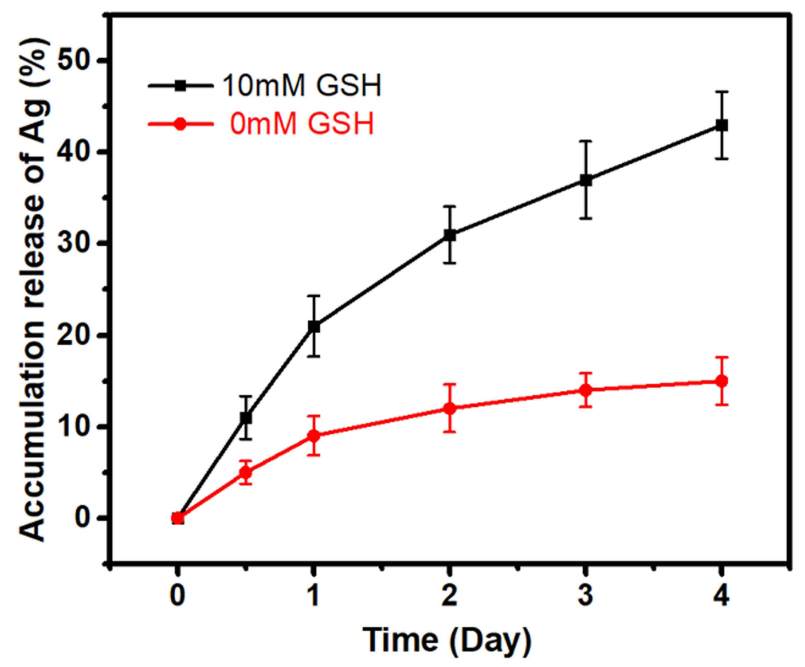

Figure 2 Drug release profiles of Ag-MONs@GEN.

Notes: (A) GEN release profiles of Ag-MONs@GEN in PBS of different GSH concentrations for 4 days. (B) Silver ion release profiles of Ag-MONs@GEN in PBS of different GSH concentrations for 4 days. Data are presented as mean value \pm SD.

Abbreviations: Ag-MONs@GEN, gentamicin-loaded nanosilver-decorated mesoporous organosilica nanoparticles; GEN, gentamicin; GSH, glutathione; SD, standard deviation. 
A
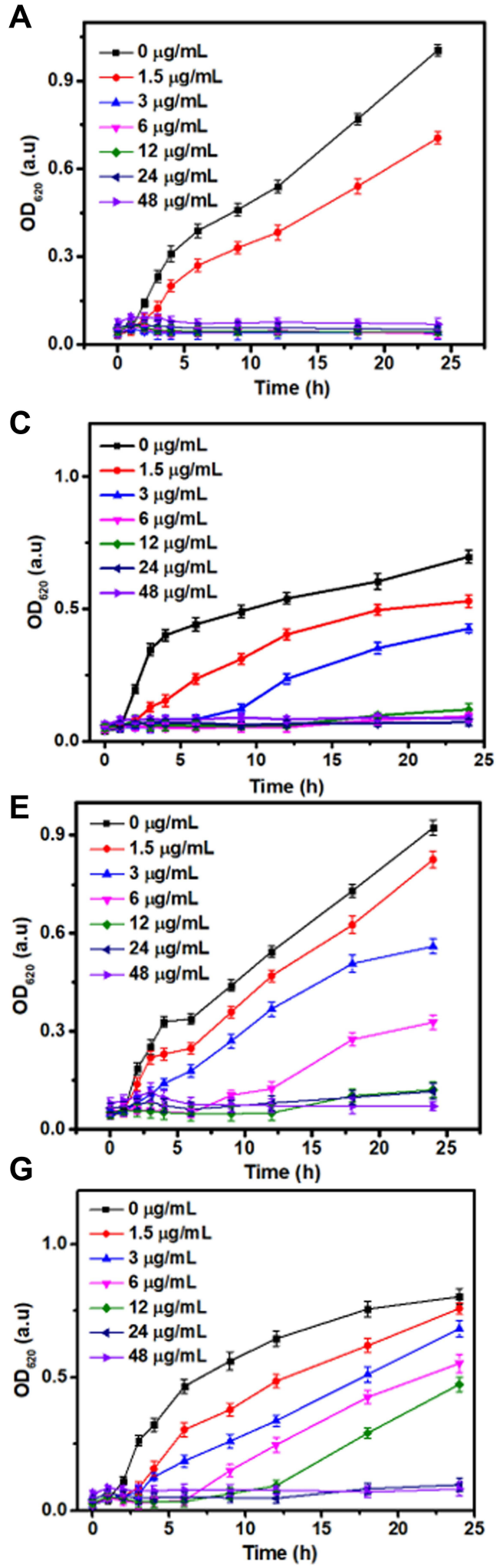

B

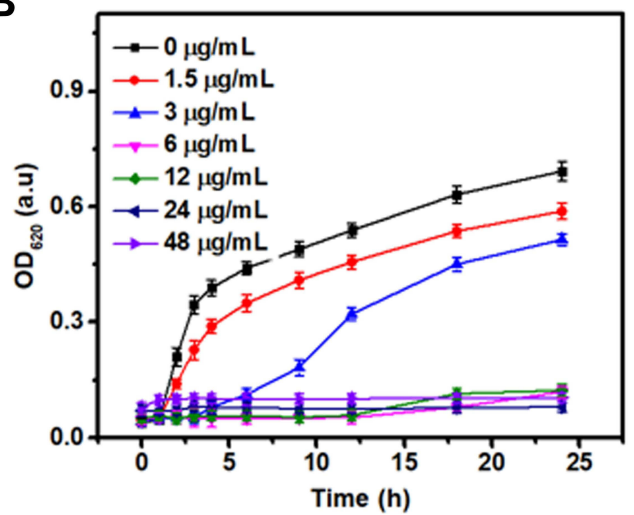

D

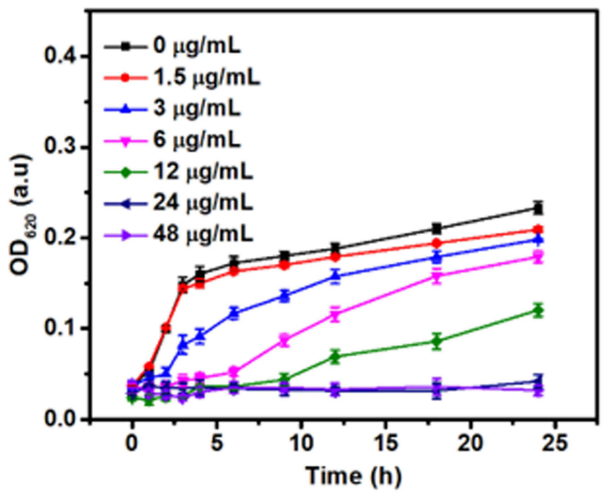

$\mathbf{F}$

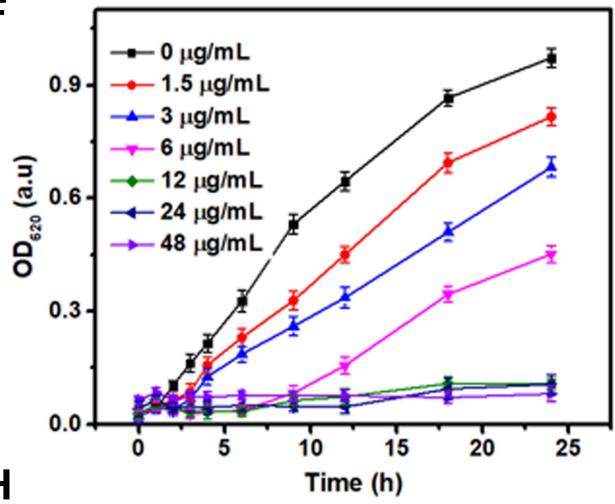

H

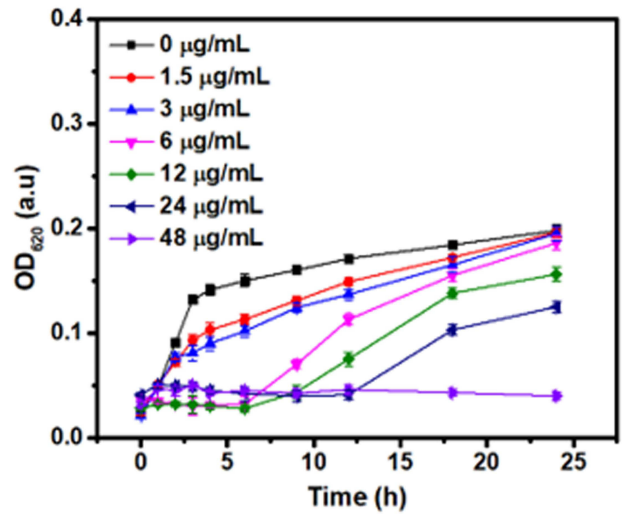

Figure 3 Bacterial growth curve of sensitive/resistant E. coli, P. aeruginosa, S. aureus, and E. faecalis with Ag-MONs@GEN at different concentrations.

Notes: $(\mathbf{A})$ sensitive E. coli. (B) sensitive P. aeruginosa. (C) sensitive S. aureus. (D) sensitive E. Faecalis. (E) resistant $E$. coli. (F) resistant $P$. aeruginosa. (G) resistant S. aureus. $(\mathbf{H})$ resistant $E$. faecalis. Data are presented as mean value $\pm S D$.

Abbreviations: Ag-MONs@GEN, gentamicin-loaded nanosilver-decorated mesoporous organosilica nanoparticles; E. coli, Escherichia coli; P. aeruginosa, Pseudomonas aeruginosa; S. aureus, Staphylococcus aureus; E. Faecalis, Enterococcus faecalis. SD, standard deviation. 
Table I MIC Values of Sensitive/Resistant E. coli, P. Aeruginosa, S. aureus, and En. faecalis with GEN, Ag-MONs, Ag-MONs@GEN

\begin{tabular}{|c|c|c|c|c|c|}
\hline \multirow[t]{2}{*}{ Bacteria } & \multicolumn{5}{|c|}{ Minimum Inhibitory Concentration (MIC) $(\mu \mathrm{g} / \mathrm{mL})$} \\
\hline & GEN & Ag-MONs(Ag) & Ag-MONs@GEN & GEN & Ag \\
\hline Sensitive E. coli & 4 & $12(0.67)$ & 3 & $(0.46)$ & $(0.17)$ \\
\hline Resistant E. coli & 128 & I $44(8.04)$ & 12 & $(1.82)$ & $(0.67)$ \\
\hline Sensitive $P$. aeruginosa & 4 & $24(1.34)$ & 6 & $(0.91)$ & $(0.33)$ \\
\hline Resistant $P$. aeruginosa & 128 & $192(10.71)$ & 12 & $(1.82)$ & $(0.67)$ \\
\hline Sensitive S. aureus & 2 & $12(0.67)$ & 6 & $(0.91)$ & $(0.33)$ \\
\hline Resistant S. aureus & 128 & I 44 (8.04) & 24 & $(3.65)$ & (1.34) \\
\hline Sensitive En. faecalis & 32 & $24(1.34)$ & 24 & $(3.65)$ & (1.34) \\
\hline Resistant En. faecalis & 256 & $192(10.71)$ & 48 & $(7.30)$ & (2.68) \\
\hline
\end{tabular}

Abbreviations: MIC, minimum inhibitory concentration; GEN, gentamicin; Ag-MONs, nanosilver-decorated mesoporous organosilica nanoparticles; Ag-MONs@GEN, gentamicin-loaded nanosilver-decorated mesoporous organosilica nanoparticles; E. coli, Escherichia coli; P. aeruginosa, Pseudomonas aeruginosa; S. aureus, Staphylococcus aureus; En. Faecalis, Enterococcus faecalis. SD, standard deviation.

Table 2 MBC Values of Sensitive/Resistant E. coli, P. aeruginosa, S. aureus, and En. faecalis with GEN, Ag-MONs, Ag-MONs@GEN

\begin{tabular}{|c|c|c|c|c|c|}
\hline \multirow[t]{2}{*}{ Bacteria } & \multicolumn{5}{|c|}{ Minimum Bactericidal Concentration (MBC) $(\mu \mathrm{g} / \mathrm{mL})$} \\
\hline & GEN & $\mathrm{Ag}-\mathrm{MONs}(\mathrm{Ag})$ & Ag-MONs@GEN & GEN & $\mathbf{A g}$ \\
\hline Sensitive $E$. coli & 8 & $12(0.67)$ & 6 & $(0.91)$ & $(0.33)$ \\
\hline Sensitive E. coli & 128 & $192(10.71)$ & 24 & $(3.65)$ & $(1.34)$ \\
\hline Sensitive $P$. aeruginosa & 8 & $48(2.68)$ & 6 & $(0.91)$ & (0.33) \\
\hline Resistant $P$. aeruginosa & 128 & $192(10.71)$ & 12 & $(1.82)$ & $(0.67)$ \\
\hline Sensitive S. aureus & 4 & $24(1.34)$ & 12 & $(1.82)$ & $(0.67)$ \\
\hline Resistant S. aureus & 128 & I 44 (8.04) & 24 & $(3.65)$ & $(1.34)$ \\
\hline Sensitive En. faecalis & 64 & $48(2.68)$ & 24 & $(3.65)$ & (1.34) \\
\hline Resistant En. faecalis & 256 & $192(|0.7|)$ & 48 & $(7.300)$ & $(2.68)$ \\
\hline
\end{tabular}

Abbreviations: MBC, minimum bactericidal concentration; GEN, gentamicin; Ag-MONs, nanosilver-decorated mesoporous organosilica nanoparticles; Ag-MONs@GEN, gentamicin-loaded nanosilver-decorated mesoporous organosilica nanoparticles; E. coli, Escherichia coli; P. aeruginosa, Pseudomonas aeruginosa; S. aureus, Staphylococcus aureus; En. Faecalis, Enterococcus faecalis. SD, standard deviation.

findings demonstrate that Ag-MONs@GEN exhibits excellent anti-bacterial effects on both susceptible and resistant bacterial strains.

We investigated bacterial colony formation of these anti-bacterial materials on LB-agar media. The corresponding MBC values of Ag-MONs@GEN, Ag-MONs, and GEN in eight bacterial strains are summarized in Table 2. For Ag-MONs@GEN, the corresponding MBC values in sensitive/resistant $E$. coli, $P$. aeruginosa, $S$. aureus, and E. faecalis were 6/24, 6/12, 12/24, and 24/ $48 \mu \mathrm{g} / \mathrm{mL}$, respectively, which was equal or higher than the corresponding MIC value. Similarly, the MBCs of AgMONs@GEN were lower than those of both Ag-MONs and GEN in all bacterial strains. To further study the excellent anti-bacterial activity of Ag-MONs@GEN, we compared the antibacterial properties of Ag-MONs@GEN with Ag-MONs, GEN, as well as the combination of AgMONs and GEN using the same silver or GEN concentration. As shown in Figures 4-7, Ag-MONs@GEN completely inhibited formation of the bacterial colonies of eight bacterial strains at each MBC concentration. However, the bacterial colonies were obvious in plates containing equal concentrations of Ag-MONs and GEN. Importantly, although the combination of Ag-MONs and GEN exhibited stronger anti-bacterial effects than AgMONs or GEN alone, their performance was weaker than Ag-MONs@GEN, indicating its synergistic antibacterial capacity. A previous study reported that GEN markedly promoted the dissolution of nanosilver, ${ }^{16}$ which not only enhanced the attachment of nanosilver onto the surface of bacteria but also facilitated the release of silver ions. Silver ions can induce the production of reactive oxygen species, ${ }^{40}$ which kill bacteria by interacting with bacterial cell walls, DNA, enzymes, and membrane proteins, possibly allowing GEN and nanosilver to play synergistic roles against resistant bacteria. To further investigate the synergistic effect, we determined the ROS production in drug-resistant E. coli after treating Ag-MON@GEN, 


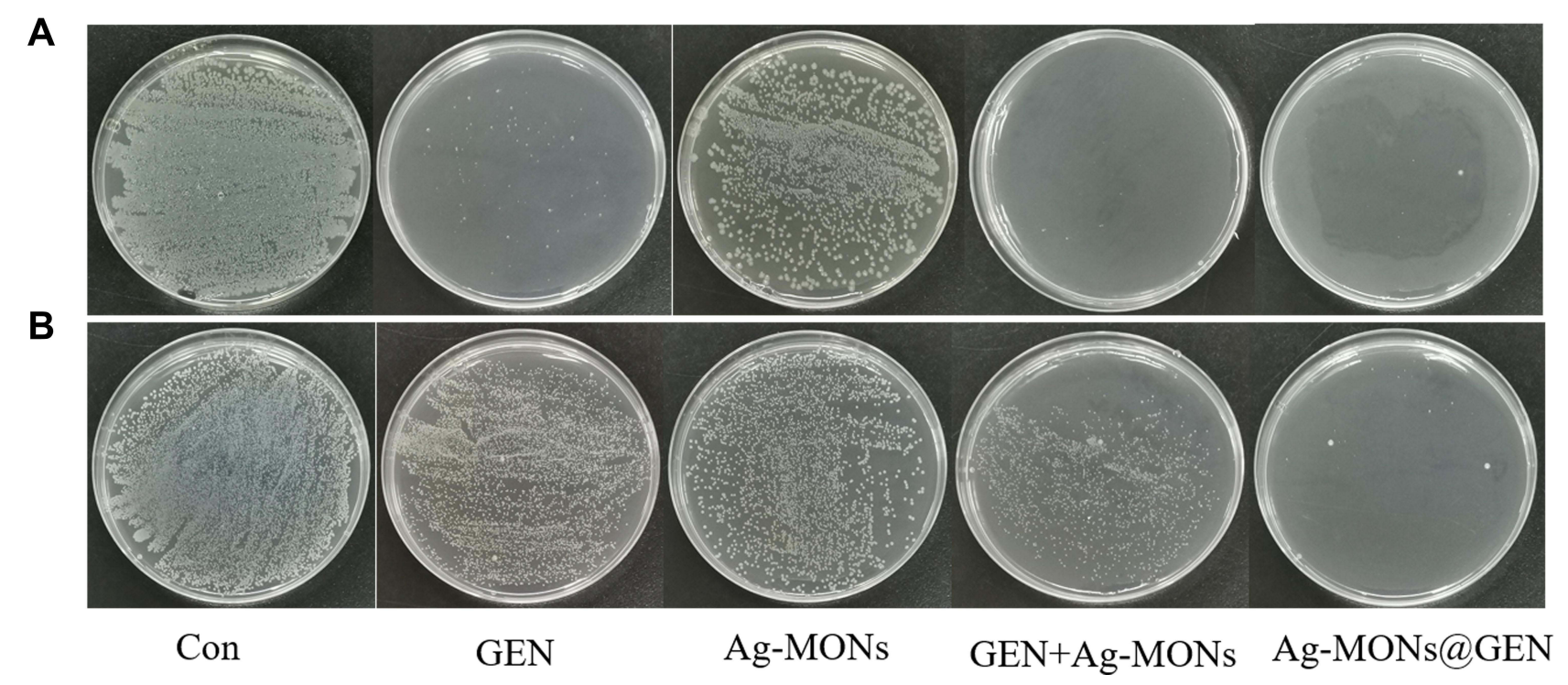

Figure 4 Synergistical antibacterial effect of Ag-MONs@GEN against sensitive/resistant $E$. coli. Notes: Photographs of LB-agar plates coated with $(\mathbf{A})$ sensitive $E$. coli. (B) resistant $E$. coli.

Abbreviations: Ag-MONs@GEN, gentamicin-loaded nanosilver-decorated mesoporous organosilica nanoparticles; E. coli, Escherichia coli; CON, control; Ag-MONs, nanosilver-decorated mesoporous organosilica nanoparticles; GEN, gentamicin; LB, lysogeny broth.

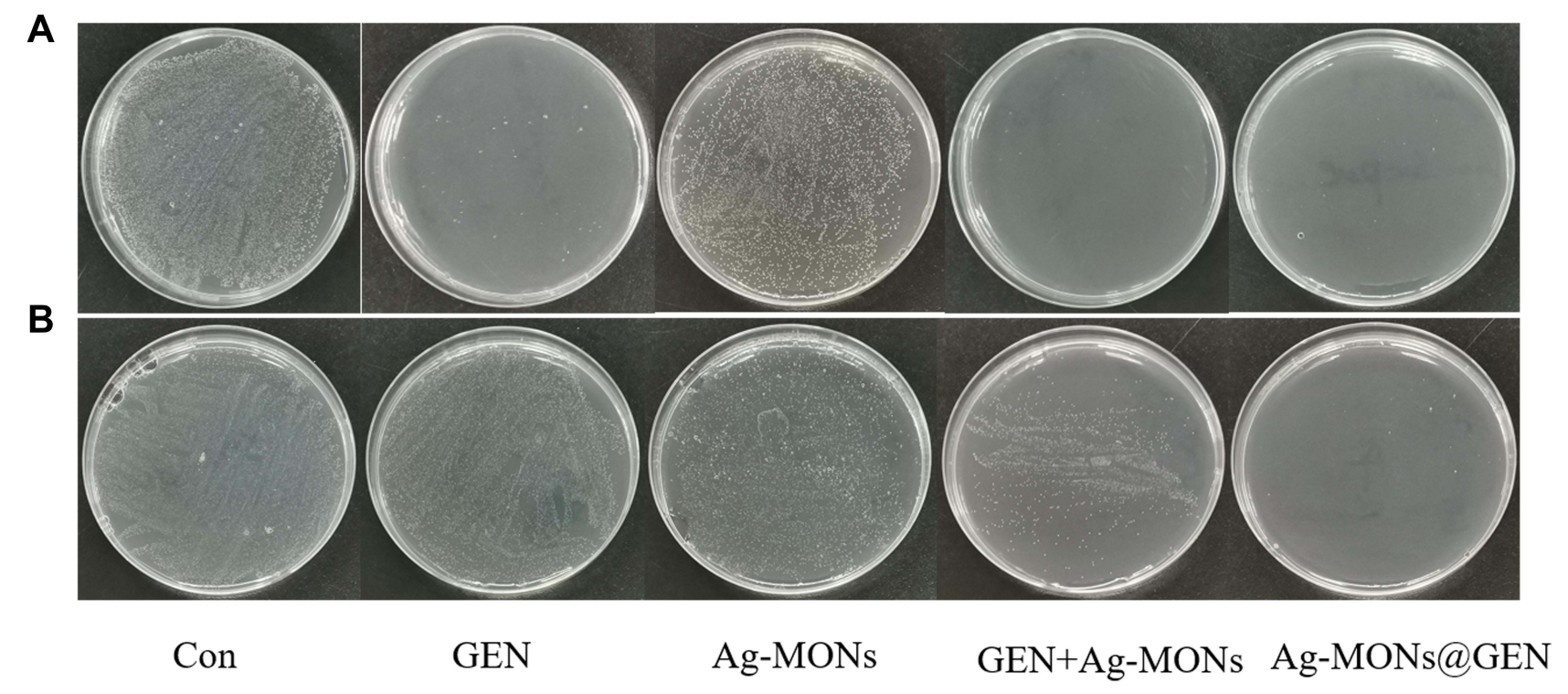

Figure 5 Synergistical antibacterial effect of Ag-MONs@GEN against sensitive/resistant $P$. aeruginosa.

Notes: Photographs of LB-agar plates coated with $(\mathbf{A})$ sensitive $P$. aeruginosa. (B) resistant $P$. aeruginosa.

Abbreviations: Ag-MONs@GEN, gentamicin-loaded nanosilver-decorated mesoporous organosilica nanoparticles; P. aeruginosa, Pseudomonas aeruginosa; CON, control; Ag-MONs, nanosilver-decorated mesoporous organosilica nanoparticles; GEN, gentamicin; LB, lysogeny broth.

Ag-MON, or GEN. As shown in Figure S4, we found that both Ag-MON and GEN induced ROS, while Ag-MON @ GEN produced the highest ROS levels. However, pretreating the ROS scavenger vitamin $\mathrm{C}$ significantly reduced the ROS generated from Ag-MON@GEN, AgMON, or GEN. Overall, these findings demonstrated that the synergistic effect of Ag-MON@GEN might be partly from the strongest ROS production in drug-resistant bacteria. We speculated that Ag-MONs@GEN might be attached to the bacterial membrane or internalized into the bacteria, and that the responsively released GEN and Ag ions might kill the bacteria. Moreover, the cationic GEN and $\mathrm{Ag}$ ions might destroy the membrane and facilitate the uptake of nanoparticles, leading to the stronger ROS production to kill bacteria. Thus, our findings suggest that the synergistic use of GEN and nanosilver in one combined 


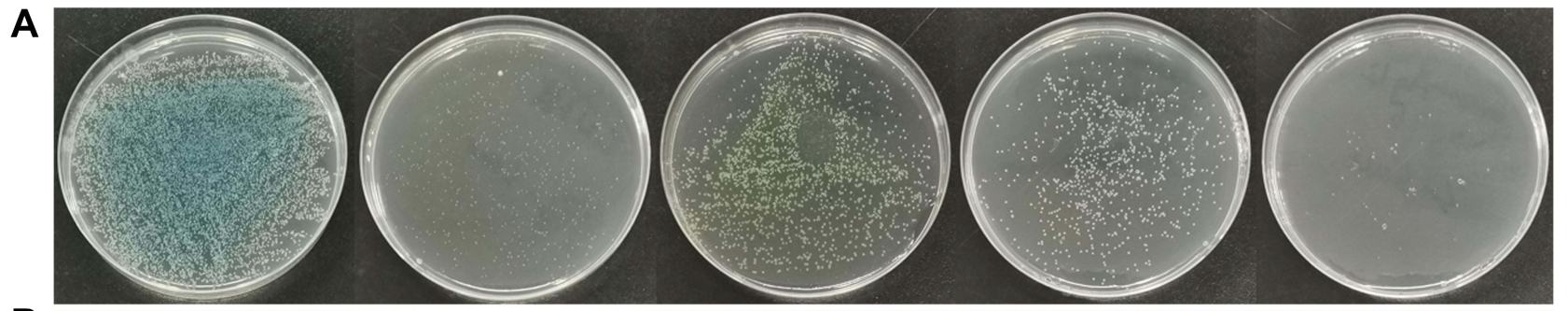

$\mathbf{B}$

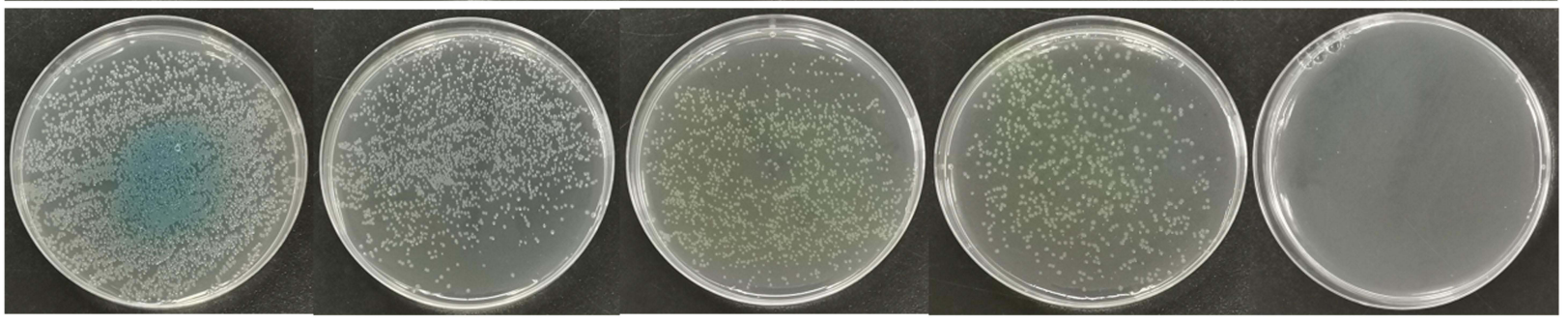

Con

GEN

Ag-MONs

GEN+Ag-MONs Ag-MONs@GEN

Figure 6 Synergistical antibacterial effect of Ag-MONs@GEN against sensitive/resistant S. aureus.

Notes: Photographs of LB-agar plates coated with (A) sensitive S. aureus. (B) resistant S. aureus.

Abbreviations: Ag-MONs@GEN, gentamicin-loaded nanosilver-decorated mesoporous organosilica nanoparticles; S. aureus, Staphylococcus aureus; CON, control; AgMONs, nanosilver-decorated mesoporous organosilica nanoparticles; GEN, gentamicin; LB, lysogeny broth.

\section{A}

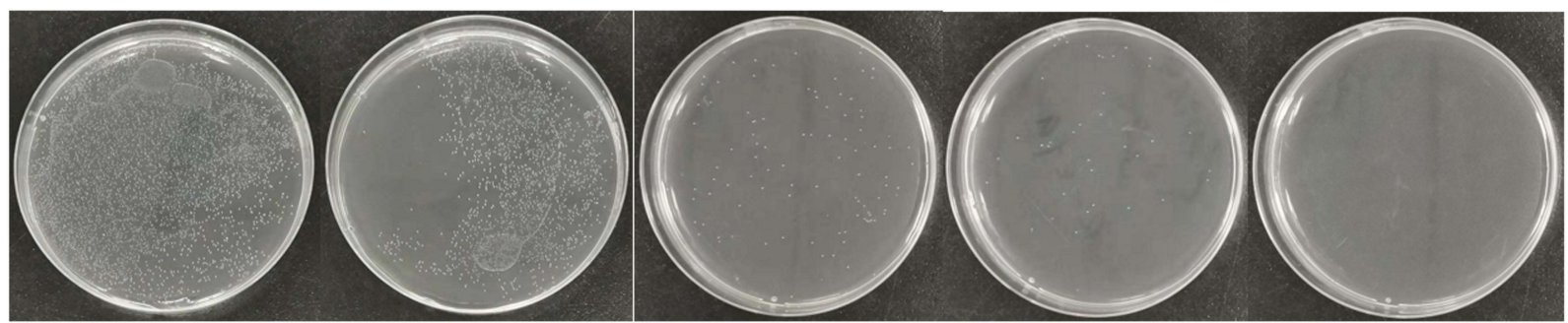

B

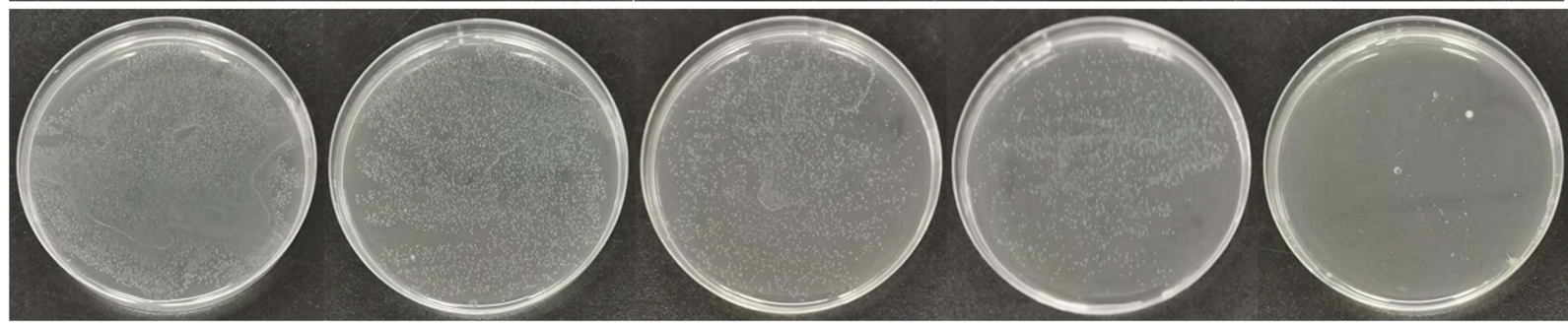

Con

GEN

Ag-MONs

GEN+Ag-MONs Ag-MONs@GEN

Figure 7 Synergistical antibacterial effect of Ag-MONs@GEN against sensitive/resistant E. faecalis.

Notes: Photographs of LB-agar plates coated with $(\mathbf{A})$ sensitive $E$. faecalis. (B) resistant $E$. faecalis.

Abbreviations: Ag-MONs@GEN, gentamicin-loaded nanosilver-decorated mesoporous organosilica nanoparticles; E. Faecalis, Enterococcus faecalis; CON, control; AgMONs, nanosilver-decorated mesoporous organosilica nanoparticles; GEN, gentamicin; LB, lysogeny broth.

platform may help combat the growing resistance against antibiotics.

In previous works, we studied the different structures and chemical components of Ag-MSNs that affected the antibacterial activity. ${ }^{20,29}$ Compared with inorganic MSNs as carriers, $\mathrm{Ag}-\mathrm{MONs}$ were degradable in response to bacterial metabolism to promote silver release and enhance antibacterial effect. $^{34}$ But it still posed a challenge to the treatment of antibiotic-resistant bacteria. Ag NP, combined with other antimicrobial agents such as antibiotics, could effectively enhance antimicrobial activity. ${ }^{41-43}$ The gentamicin promoted the interaction of silver and bacteria, indicating synergistic antibacterial. ${ }^{16}$ The large surface area of Ag-MONs was conducive to the 
co-delivery of gentamicin, thus facilitating safer and synergistic therapy. Our results demonstrated that AgMONs@GEN had synergistic antibacterial effects and reversed the antibiotic tolerance of bacteria. The synergistic antimicrobial strategy significantly reduced the concentration of drug administration, expanded the safety window of drug administration, and improved the biosafety. However, antibacterial efficacy and biosafety need to be further validated in vivo. More efforts are needed to improve the bioavailability of silver nanoparticles and develop simpler synthesis methods for large-scale production and achieve broad-spectrum antimicrobial activity.

In light of the important impact of toxicology on the future clinical application of nanomedicine, we evaluated the effects of anti-bacterial agent exposure on normal cell growth. We incubated L929 and HUVECs with Ag-MONs@GEN, Ag-MONs, and GEN for 24 h, after which the cell viability was determined. As shown in Figure 8 and S5, GEN had no significant toxic effects on cells, while Ag-MONs@GEN and Ag-MONs showed similar dose-dependent cytotoxic profile. The $\mathrm{IC}_{50}$ values of Ag-MONs@GEN in L929, HUVECs, and Raw 264.7 were $313.6 \pm 15.9,295.7 \pm 12.3$, and 192.5 $\pm 16.8 \mu \mathrm{g} / \mathrm{mL}$, respectively, which were much higher than their corresponding MIC or MBC value. Our previous reports showed that the $\mathrm{IC}_{50}$ values of $\mathrm{Ag}-\mathrm{MONs}$ were $247.6 \pm 3.799 \mu \mathrm{g} / \mathrm{mL}$ on HUVECs, and $328.2 \pm$ $5.252 \mu \mathrm{g} / \mathrm{mL}$ on L929 cells. $^{39}$ The similar toxicities indicate there was less difference of Ag-MONs after loading GEN, suggesting its good biocompatibility on the therapeutic dose.

\section{Conclusions}

In summary, biodegradable nanosilver-decorated MONs were fabricated for delivery GEN to achieve synergetic effects against antibiotic-resistant bacteria. The GSHresponsive matrix degradation of Ag-MONs@GEN endowed the controlled release of GEN and silver ions simultaneously. The nanoplatform of Ag-MONs@GEN exhibited synergistic antibacterial activity to Ag-MONs and GEN alone via inducing ROS generation, especially on four antibiotic-resistant bacteria including $E$. coli, P. aeruginosa, S. aureus, and E. faecalis. Furthermore, AgMONs@GEN exhibited good biocompatibility in L929, HUVECs, and Raw 264.7 cells. Our findings demonstrate that the integration of nanosilver and GEN into one biodegradable nanoplatform has synergetic and safe effects against antibiotic-resistant bacteria. With these abovementioned advantages, we believe that the Ag-MONs@GEN developed in this work will have promising effects against bacteria, particularly antibiotic-resistant bacteria. Gentamicin, as a commonly used antibiotic, can also promote the introduction of silver ions into bacteria. We speculate that the simultaneous delivery of gentamicin and nanosilver can enhance the antibacterial ability and kill antibiotic-resistant bacteria.
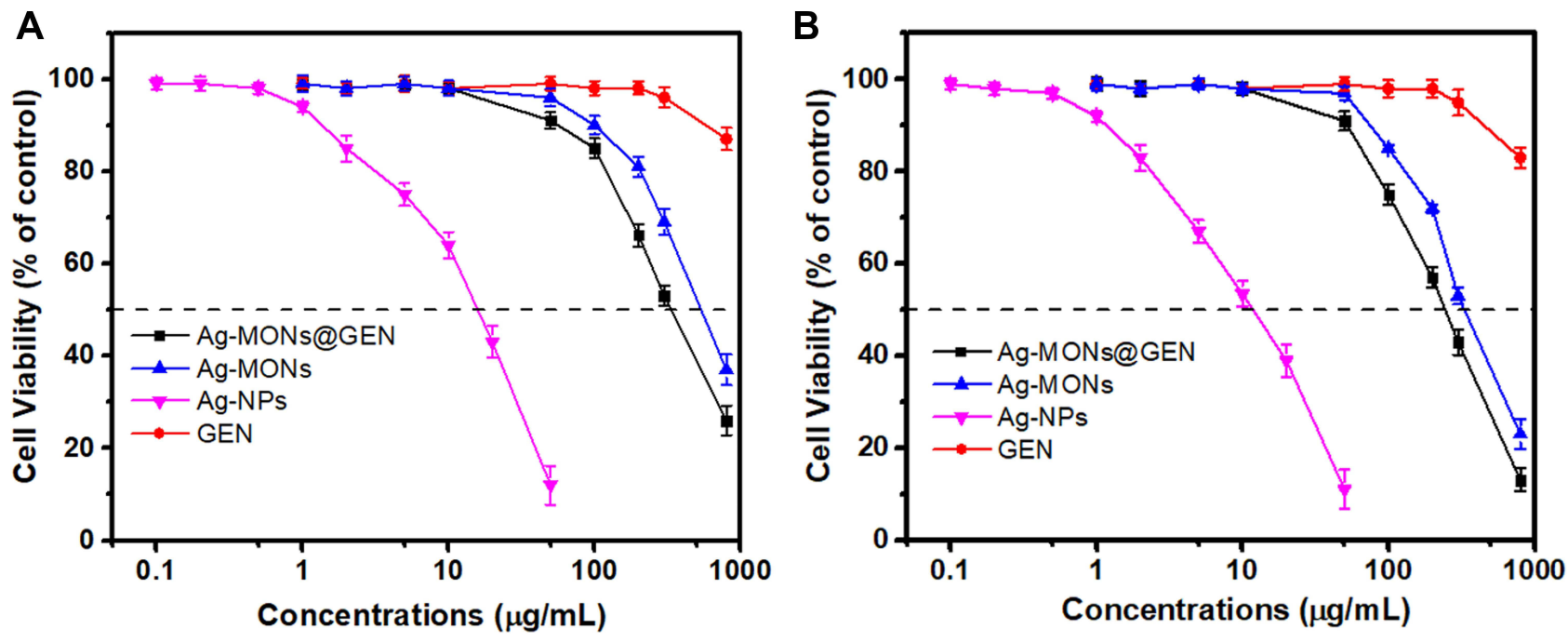

Figure 8 Cytotoxicity of Ag-NPs, GEN, Ag-MONs, or Ag-MONs@GEN.

Notes: Cell viabilities of (A) L929 and (B) HUVECs cells after treating Ag-NPs, GEN, Ag-MONs, or Ag-MONs@GEN at different concentrations for 24 h. Data are presented as mean value \pm SD.

Abbreviations: Ag-NPs, nanosilver; GEN, gentamicin; Ag-MONs, nanosilver-decorated mesoporous organosilica nanoparticles; Ag-MONs@GEN, gentamicin-loaded nanosilver-decorated mesoporous organosilica nanoparticles; HUVEC, human umbilical vein endothelial cell; SD, standard deviation. 


\section{Acknowledgments}

This study was supported by the Henan Science and Technology Research Project NO.192102310053 and China Postdoctoral Science Foundation (NO. 2020M672641).

\section{Disclosure}

The authors report no conflicts of interest in this work.

\section{References}

1. Arias CA, Murray BE. Antibiotic-resistant bugs in the 21 st century--a clinical super-challenge. $N$ Engl J Med. 2009;360(5):439-443. doi:10.1056/NEJMp0804651

2. Baur D, Gladstone BP, Burkert F, et al. Effect of antibiotic stewardship on the incidence of infection and colonisation with antibiotic-resistant bacteria and Clostridium difficile infection: a systematic review and meta-analysis. Lancet Infect Dis. 2017;17 (9):990-1001. doi:10.1016/s1473-3099(17)30325-0

3. Tacconelli E, Carrara E, Savoldi A, et al. Discovery, research, and development of new antibiotics: the WHO priority list of antibiotic-resistant bacteria and tuberculosis. Lancet Infect Dis. 2018;18(3):318-327. doi:10.1016/S1473-3099(17)30753-3

4. Gupta A, Mumtaz S, Li CH, Hussain I, Rotello VM. Combatting antibiotic-resistant bacteria using nanomaterials. Chem Soc Rev 2019;48(2):415-427. doi:10.1039/C7CS00748E

5. Makabenta JMV, Nabawy A, Li CH, Schmidt-Malan S, Patel R, Rotello VM. Nanomaterial-based therapeutics for antibiotic-resistant bacterial infections. Nat Rev Microbiol. 2021;19(1):23-36. doi:10. 1038/s41579-020-0420-1

6. Lee MMS, Xu W, Zheng L, et al. Ultrafast discrimination of Gram-positive bacteria and highly efficient photodynamic antibacterial therapy using near-infrared photosensitizer with aggregation-induced emission characteristics. Biomaterials. 2020;230:119582. doi:10.1016/ j.biomaterials.2019.119582

7. Liu Z, Wang F, Ren J, Qu X. A series of MOF/Ce-based nanozymes with dual enzyme-like activity disrupting biofilms and hindering recolonization of bacteria. Biomaterials. 2019;208:21-31. doi:10. 1016/j.biomaterials.2019.04.007

8. Sun Y, Sun X, Li X, et al. A versatile nanocomposite based on nanoceria for antibacterial enhancement and protection from aPDT-aggravated inflammation via modulation of macrophage polarization. Biomaterials. 2021;268:120614. doi:10.1016/j.biomaterials.2020.120614

9. Rizzello L, Pompa PP. Nanosilver-based antibacterial drugs and devices: mechanisms, methodological drawbacks, and guidelines. Chem Soc Rev. 2014;43(5):1501-1518. doi:10.1039/C3CS60218D

10. Deshmukh SP, Patil SM, Mullani SB, Delekar SD. Silver nanoparticles as an effective disinfectant: a review. Mater Sci Eng C Mater Biol Appl. 2019;97:954-965. doi:10.1016/j.msec.2018.12.102

11. Long YM, Hu LG, Yan XT, et al. Surface ligand controls silver ion release of nanosilver and its antibacterial activity against Escherichia coli. Int J Nanomed. 2017;12:3193-3206. doi:10.2147/IJN.S132327

12. Poustchi F, Amani H, Ahmadian Z, et al. Combination therapy of killing diseases by injectable hydrogels: from concept to medical applications. Adv Healthc Mater. 2021;10:2001571. doi:10.1002/ adhm.202001571

13. Hwang IS, Hwang JH, Choi H, Kim KJ, Lee DG. Synergistic effects between silver nanoparticles and antibiotics and the mechanisms involved. $J$ Med Microbiol. 2012;61(Pt 12):1719-1726. doi:10.1099/jmm.0.047100-0

14. Wang MZ, Sun HY, Long X, Lin ZF. Combined Antibacterial Property and Mechanism of Nanosilver Composites and Antibiotics against Bacteria. Asian J Ecotoxicol. 2020;2:39-49. doi:10.7524/ AJE.1673-5897.20191204001
15. Abdel Rahim KA, Ali Mohamed AM. Bactericidal and Antibiotic Synergistic Effect of Nanosilver Against Methicillin-Resistant Staphylococcus aureus. Jundishapur J Micro. 2015;8(11):e25867. doi:10.5812/jjm.25867

16. Wang YW, Tang H, Wu D, et al. Enhanced bactericidal toxicity of silver nanoparticles by the antibiotic gentamicin. Environ Sci-Nano. 2016;3(4):788-798. doi:10.1039/C6EN00031B

17. Katva S, Das S, Moti HS, Jyoti A, Kaushik S. Antibacterial Synergy of Silver Nanoparticles with Gentamicin and Chloramphenicol against Enterococcus faecalis. Pharmacogn Mag. 2018;13(Suppl 4): S828-S33. doi:10.4103/pm.pm-120-17

18. Mazur P, Skiba-Kurek I, Mrowiec P, Karczewska E, Drozdz R. Synergistic ROS-Associated Antimicrobial Activity of Silver Nanoparticles and Gentamicin Against Staphylococcus epidermidis. Int J Nanomed. 2020;15:3551-3562. doi:10.2147/IJN.S246484

19. Zhu Y, Xu J, Wang Y, et al. Silver nanoparticles-decorated and mesoporous silica coated single-walled carbon nanotubes with an enhanced antibacterial activity for killing drug-resistant bacteria. Nano Res. 2020;13(2):389-400. doi:10.1007/s12274-020-2621-3

20. Chang ZM, Wang Z, Lu MM, et al. Janus silver mesoporous silica nanobullets with synergistic antibacterial functions. Colloid Surface B. 2017;157:199-206. doi:10.1016/j.colsurfb.2017.05.079

21. Lu MM, Wang QJ, Chang ZM, et al. Synergistic bactericidal activity of chlorhexidine-loaded, silver-decorated mesoporous silica nanoparticles. Int J Nanomed. 2017;12:3577-3589. doi:10.2147/IJN.S133846

22. Wang Y, Ding X, Chen Y, et al. Antibiotic-loaded, silver core-embedded mesoporous silica nanovehicles as a synergistic antibacterial agent for the treatment of drug-resistant infections. Biomaterials. 2016;10 1:207-216. doi:10.1016/j.biomaterials.2016.06.004

23. Wang X, Sun W, Yang W, Gao S, Sun C, Li Q. Mesoporous silica-protected silver nanoparticle disinfectant with controlled Ag+ ion release, efficient magnetic separation, and effective antibacterial activity. Nano Adv. 2019;1(2):840-848. doi:10.1039/C8NA00275D

24. Lu MM, Bai J, Shao D, et al. Antibacterial and biodegradable tissue nano-adhesives for rapid wound closure. Int $J$ Nanomed. 2018;13:5849-5863. doi:10.2147/IJN.S177109

25. Wan X, Wu L, Pei H, Ke H, Yang G, Tang J. Surface functionality density regulated in situ reduction of nanosilver on hierarchial wrinkled mesoporous silica nanoparticles and their antibacterial activity. RSC Adv. 2018;8(35):19420-19425. doi:10.1039/C8RA02903B

26. Hasanzadeh A, Gholipour B, Rostamnia S, et al. Biosynthesis of AgNPs onto the urea-based periodic mesoporous organosilica (AgxNPs/ Ur-PMO) for antibacterial and cell viability assay. J Colloid Interface Sci. 2021;585:676-683. doi:10.1016/j.jcis.2020.10.047

27. Liu J, Li S, Fang Y, Zhu Z. Boosting antibacterial activity with mesoporous silica nanoparticles supported silver nanoclusters. J Colloid Interface Sci. 2019;555:470-479. doi:10.1016/j.jcis.2019.08.009

28. Du X, Li X, Xiong L, Zhang X, Kleitz F, Qiao SZ. Mesoporous silica nanoparticles with organo-bridged silsesquioxane framework as innovative platforms for bioimaging and therapeutic agent delivery. Biomaterials. 2016;91:90-127. doi:10.1016/j.biomaterials.2016.03.019

29. Lu MM, Ge Y, Qiu J, et al. Redox/pH dual-controlled release of chlorhexidine and silver ions from biodegradable mesoporous silica nanoparticles against oral biofilms. Int $J$ Nanomed. 2018;13:7697-7709. doi:10.2147/IJN.S181168

30. Shao D, Li M, Wang Z, et al. Bioinspired Diselenide-Bridged Mesoporous Silica Nanoparticles for Dual-Responsive Protein Delivery. Adv Mater. 2018;30(29):e1801198. doi:10.1002/adma.201801198

31. Shao D, Zhang F, Chen F, et al. Biomimetic Diselenide-Bridged Mesoporous Organosilica Nanoparticles as an X-ray-Responsive Biodegradable Carrier for Chemo-Immunotherapy. Adv Mater. 2020;32(50):e2004385. doi:10.1002/adma.202004385

32. He Y, Zhang Y, Sun M, et al. One-pot synthesis of chlorhexidine-templated biodegradable mesoporous organosilica nanoantiseptics. Colloid Surface B. 2020;187:110653. doi:10.1016/j. colsurfb.2019.110653 
33. Wang Z, Zhang F, Shao D, et al. Janus Nanobullets Combine Photodynamic Therapy and Magnetic Hyperthermia to Potentiate Synergetic Anti-Metastatic Immunotherapy. Adv Sci. 2019;6 (22):1901690. doi:10.1002/advs.201901690

34. Gao F, Wu J, Niu S, et al. Biodegradable, pH-Sensitive Hollow Mesoporous Organosilica Nanoparticle (HMON) with Controlled Release of Pirfenidone and Ultrasound-Target-MicrobubbleDestruction (UTMD) for Pancreatic Cancer Treatment. Theranostics. 2019;9(20):6002-6018. doi:10.7150/thno.36135

35. Cheng Y, Jiao X, Fan W, Yang Z, Wen Y, Chen X. Controllable synthesis of versatile mesoporous organosilica nanoparticles as precision cancer theranostics. Biomaterials. 2020;256:120191. doi:10.1016/j.biomaterials.2020.120191

36. Shao D, Wang Z, Chang Z, Chen L, Dong WF. Janus Metallic Mesoporous Silica Nanoparticles: unique Structures for Cancer. Curr Opin Biothch. 2021;100294. doi:10.1016/j.cobme.2021.100294

37. Hu H, Yang C, Zhang F, et al. Robust Platform for the Scalable Manufacture of Biomimetic Nanovaccines. Adv Sci. 2021;2002020. doi:10.1002/advs.202002020

38. Dawulieti J, Sun M, Zhao Y. Treatment of severe sepsis with nanoparticulate cell-free DNA scavengers. Adv Sci. 2020;6(22):eaay7148. doi:10.1126/sciadv.aay7148
39. Zhang Y, He Y, Shi C, et al. Tannic Acid-Assisted Synthesis of Biodegradable and Antibacterial Mesoporous Organosilica Nanoparticles Decorated with Nanosilver. ACS Sustain Chem Eng. 2020;8(3):1695-1702. doi:10.1021/acssuschemeng.9b07576

40. Park HJ, Kim JY, Kim J, et al. Silver-ion-mediated reactive oxygen species generation affecting bactericidal activity. Water Res. 2009;43 (4):1027-1032. doi:10.1016/j.watres.2008.12.002

41. Giedraitienè A, Vitkauskienė A, Naginienė R, Pavilonis A. Antibiotic Resistance Mechanisms of Clinically Important Bacteria. Medicina. 2011;47(3):19. doi:10.3390/medicina47030019

42. Liu R, Wang X, Ye J, et al. Enhanced antibacterial activity of silver-decorated sandwich-like mesoporous silica/reduced graphene oxide nanosheets through photothermal effect. Nanotechnology. 2018;29(10):105704. doi:10.1088/1361-6528/aaa624

43. Shi L, Zhang W, Yang K, et al. Antibacterial and osteoinductive capability of orthopedic materials via cation $-\pi$ interaction mediated positive charge. J Mater Chem B. 2015;3(5):733-737. doi:10.1039/ c4tb01924e
International Journal of Nanomedicine

\section{Publish your work in this journal}

The International Journal of Nanomedicine is an international, peerreviewed journal focusing on the application of nanotechnology in diagnostics, therapeutics, and drug delivery systems throughout the biomedical field. This journal is indexed on PubMed Central, MedLine, CAS, SciSearch ${ }^{\mathbb{}}$, Current Contents ${ }^{\mathbb{R}} /$ Clinical Medicine, $^{2}$

\section{Dovepress}

Journal Citation Reports/Science Edition, EMBase, Scopus and the Elsevier Bibliographic databases. The manuscript management system is completely online and includes a very quick and fair peer-review system, which is all easy to use. Visit http://www.dovepress.com/ testimonials.php to read real quotes from published authors. 